\title{
RESEARCH HIGHLIGHT OPEN H3.3S31 phosphorylation: linking transcription elongation to stimulation responses
}

\author{
Hong Wen ${ }^{1}$ and Xiaobing Shi ${ }^{1}$ \\ Signal Transduction and Targeted Therapy (2020)5:176
}

; https://doi.org/10.1038/s41392-020-00293-6
Phosphorylation of histone proteins is involved in multiple cellular functions such as mitosis, DNA damage response, and transcriptional regulation. For example, phosphorylation of $\mathrm{H} 3.3$ Ser31 (H3.3S31 ph) is known as a mitotic epigenetic modification. ${ }^{1}$ In a recent paper published on Nature, Armache et al. show that $\mathrm{H} 3.3 \mathrm{~S} 31 \mathrm{ph}$ has an unexpected function in stimulation-induced transcription through crosstalk with $\mathrm{H} 3 \mathrm{~K} 36 \mathrm{me} 3$, an epigenetic mark for transcription elongation. ${ }^{2}$ In eukaryotic cells, DNA is wrapped around histone octamers, which contain two copies of each of the core histones $\mathrm{H} 2 \mathrm{~A}, \mathrm{H} 2 \mathrm{~B}, \mathrm{H} 3$, and $\mathrm{H} 4$. Most histones, also known as canonical histones, are synthesized and deposited onto chromatin only during $S$ phase when DNA is being replicated. There are also other histones, known as histone variants, that are expressed and synthesized in a cell cycleindependent manner. Incorporation of histone variants into nucleosomes provides a means to regulate the chromatin architecture in addition to other epigenetic mechanisms. Histone variants differ from canonical histones in just a few amino acids or large fractions of polypeptides. One of the histone variants, H3.3, has only four- or five-amino acid residue difference from canonical H3, namely H3.1 and H3.2, respectively. Three amino acids are located in the histone core domain important for binding to H3.3specific histone chaperones. The only different residue at the $\mathrm{N}$ terminus of the protein is at position 31, which is an Alanine in $\mathrm{H} 3.1$ and H3.2 but replaced by highly conserved Serine in H3.3. Phosphorylation of H3.3S31 was first reported 15 years ago, ${ }^{1}$ however, the function of this modification remains largely unknown.

By mass spectrometric analysis of purified histones from primary mouse bone marrow-derived macrophages (BMDMs), Armache et al. detected an increase of H3.3S31ph in lipopolysaccharide (LPS)-stimulated cells. Using a homemade H3.3S31 ph specific antibody, they further corroborated that $\mathrm{H} 3.3 \mathrm{~S} 31 \mathrm{ph}$ is a stimulation-associated histone modification in four additional cell types responding to different physiological stimuli. Chromatin immunoprecipitation followed by high-throughput sequencing (ChIP-seq) analysis revealed an enrichment of $\mathrm{H} 3.3531$ ph specifically in LPS stimulation-induced genes. The enrichment of $\mathrm{H} 3.3 \mathrm{~S} 31 \mathrm{ph}$ in gene bodies was abolished or significantly reduced by treating the cells with P-TEFb inhibitor flavopiridol or TOP1 inhibitor camptothecin, both of which block the elongation of RNA polymerase II (Pol II), suggesting a direct link between $\mathrm{H} 3.3 \mathrm{~S} 31 \mathrm{ph}$ and transcription elongation. Previous studies described that several kinases, such as IKKa, CHK1, and aurora B, are able to phosphorylate $\mathrm{H} 3.3 \mathrm{~S} 31$. A series of genetic perturbation using short hairpin RNA (shRNA) and pharmacological inhibition experiments suggested that IKKa is likely responsible for the deposition of co-transcriptional H3.3S31ph at inflammatory genes.

The distribution pattern of $\mathrm{H} 3.3 \mathrm{S3} 1 \mathrm{ph}$ in gene body is reminiscent of that of another transcription elongationassociated histone modification, H3K36me3. H3K36me3 is mediated by a single histone methyltransferase, SETD2. Given this link between $\mathrm{H} 3.3 \mathrm{~S} 31 \mathrm{ph}$ and $\mathrm{H} 3 \mathrm{~K} 36 \mathrm{me} 3$ as well as their physical proximity on the H3.3 tail, Armache et al. hypothesized that H3.3S31ph may provide stimulation-induced genes with the capacity for augmented transcription, in part through the stimulation of H3K36me3. Indeed, the crystal structure of the human SETD2 catalytic domain bound to a H3.3 peptide bearing $\mathrm{S} 31 \mathrm{ph}$ and related biochemical data supported this hypothesis. Two basic residues of SETD2, K1600, and K1673, provide positive charge recognizing the phosphorylated H3.3S31, thus promoting the engagement of $\mathrm{H} 3.3 \mathrm{~K} 36$ at the catalytic site for methylation. Consistent with the structural observation, SETD2 showed higher catalytic activity on the phosphomimic H3.3S31E-containing nucleosomes over unmodified nucleosomes; however, the stimulatory effect was lost by mutating any of the two basic lysine residues to glutamine (K1600E or K1673E). Together, these data suggest that IKKa stimulates target gene expression by H3.3S31ph-medaited augmentation of SETD2 activity.

Previously, we and others identified ZMYND11 as an H3.3K36me3 specific reader that functions as a transcriptional corepressor and tumor suppressor. ${ }^{3}$ In addition to K36me3, unmodified S31 also contributes to the binding. Structural and biochemical data suggested that a phosphate at Ser31 impedes ZMYND11 binding to H3.3K36me3. Consistent with these in vitro data, inflammatory genes tend to have pre-existing H3.3K36me3 with coincident ZMYND11 occupancy in resting BMDMs; whereas upon stimulation, ZMYND11 is rejected from these genes. This is likely a consequence of IKKa-mediated H3.3S31 ph, as ZMYND11 is retained at inflammatory genes in cells treated with an IKK inhibitor before LPS stimulation. Finally, to directly define the function of H3.3531ph in inflammatory gene induction, Armache et al. generated H3f3a H3f3b double knockout (DKO) RAW264.7 macrophage cell lines using CRISPR-Cas9 and rescued the DKO cells with the wild-type, H3.3S31A (loss of function) and H3.3S31E (gain of function, phosphomimic) transgenes. Overall, LPS-induced gene expression in H3.3 DKO macrophages was increased by wildtype H3.3 or H3.3S31E transduction, but not by the H3.3S31A mutant, suggesting an essential role of $\mathrm{H} 3.3 \mathrm{~S} 31$ ph in promoting rapid stimulation-induced transcription in macrophage-like cell lines.

\footnotetext{
${ }^{1}$ Center for Epigenetics, Van Andel Institute, Grand Rapids, MI 49503, USA
}

Correspondence: Xiaobing Shi (Xiaobing.shi@vai.org)

Received: 27 July 2020 Revised: 13 August 2020 Accepted: 17 August 2020

Published online: 29 August 2020 


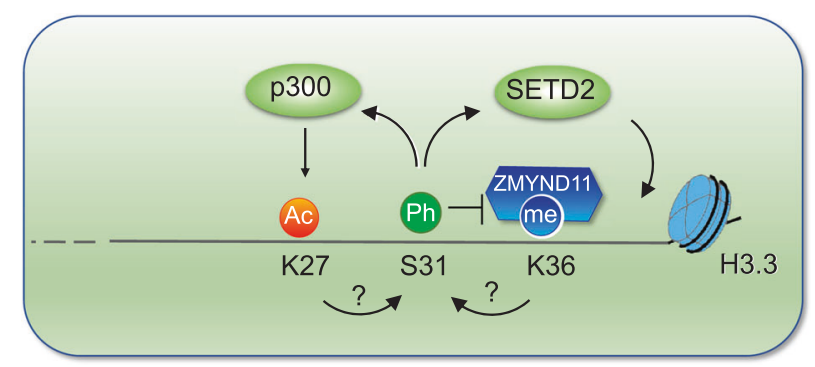

Fig. 1 Crosstalk between variant-specific phosphorylation on H3.3S31 and other modifications. H3.3S31ph promotes SETD2dependent trimethylation on $\mathrm{H} 3 \mathrm{~K} 36$ and inhibits binding of H3K36me3 by the transcription corepressor ZMYND11, thus enabling rapid and robust gene expression. $\mathrm{H} 3.3 \mathrm{~S} 31 \mathrm{ph}$ can also enhance the enzymatic activity of the histone acetyltransferase p300 to promote transcription by H3K27 acetylation. Ac acetylation. Me methylation. $\mathrm{Ph}$ phosphorylation

Despite that $\mathrm{H} 3.3531 \mathrm{ph}$ was first reported as a mitotic epigenetic modification 15 years ago, ${ }^{1}$ its function in interphase remained unknown. Studies from two laboratories recently showed that $\mathrm{H} 3.3 \mathrm{~S} 31$ ph stimulates the catalytic activity of histone acetyltransferase p300, thus maintaining enhancers at an open, acetylated chromatin state permissive to the embryonic development program. ${ }^{4,5}$ In the current study, Armache et al. ${ }^{2}$ discovered a novel role of $\mathrm{H} 3.3 \mathrm{~S} 31 \mathrm{ph}$ in transcription elongation. It not only augments the catalytic activity of SETD2, but also ejects negative regulator ZMYND11. Thus, together with $\mathrm{H} 3.3 \mathrm{~K} 36 \mathrm{me} 3, \mathrm{H} 3.3 \mathrm{~S} 31 \mathrm{ph}$ provides an epigenetic signature in gene bodies promoting rapid and high-level expression of stimulation-induced genes (Fig. 1). The region of histone $\mathrm{H} 3$ tail between $\mathrm{K} 27$ to $\mathrm{K} 36$ has become a research focus in the chromatin field, in part because of frequent mutations of histone H3K27, G34, and $\mathrm{K} 36$ in various pediatric tumors. Interestingly, many of these mutations occur in H3.3encoding genes. Given the importance of the crosstalk between $\mathrm{H} 3.3 \mathrm{~S} 31 \mathrm{ph}$ and modifications at neighboring residues in transcription regulation, it would be interesting to determine in future studies the role of $\mathrm{H} 3.3 \mathrm{~S} 31 \mathrm{ph}$ in the pediatric tumors bearing the deadly histone mutations.

\section{ACKNOWLEDGEMENTS}

This work was supported in part by grants from NCI (CA204020) and Leukaemia \& Lymphoma Society Career Development Program (1339-17) to X.S.

\section{ADDITIONAL INFORMATION}

Competing interests: X.S. is a SAB member of EpiCypher. Other authors declare no competing interests.

\section{REFERENCES}

1. Hake, S. B. et al. Serine 31 phosphorylation of histone variant H3.3 is specific to regions bordering centromeres in metaphase chromosomes. Proc. Natl Acad. Sci. USA 102, 6344-6349 (2005).

2. Armache, A. et al. Histone H3.3 phosphorylation amplifies stimulation-induced transcription. Nature 583, 852-857 (2020).

3. Wen, H. et al. ZMYND11 links histone H3.3K36me3 to transcription elongation and tumour suppression. Nature 508, 263-268 (2014).

4. Martire, S. et al. Phosphorylation of histone H3.3 at serine 31 promotes p300 activity and enhancer acetylation. Nat. Genet. 51, 941-946 (2019).

5. Sitbon, D. et al. Histone variant H3.3 residue S31 is essential for Xenopus gastrulation regardless of the deposition pathway. Nat. Commun. 11, 1256 (2020).

(i) Open Access This article is licensed under a Creative Commons c. Attribution 4.0 International License, which permits use, sharing, adaptation, distribution and reproduction in any medium or format, as long as you give appropriate credit to the original author(s) and the source, provide a link to the Creative Commons license, and indicate if changes were made. The images or other third party material in this article are included in the article's Creative Commons license, unless indicated otherwise in a credit line to the material. If material is not included in the article's Creative Commons license and your intended use is not permitted by statutory regulation or exceeds the permitted use, you will need to obtain permission directly from the copyright holder. To view a copy of this license, visit http://creativecommons. org/licenses/by/4.0/.

(c) The Author(s) 2020 